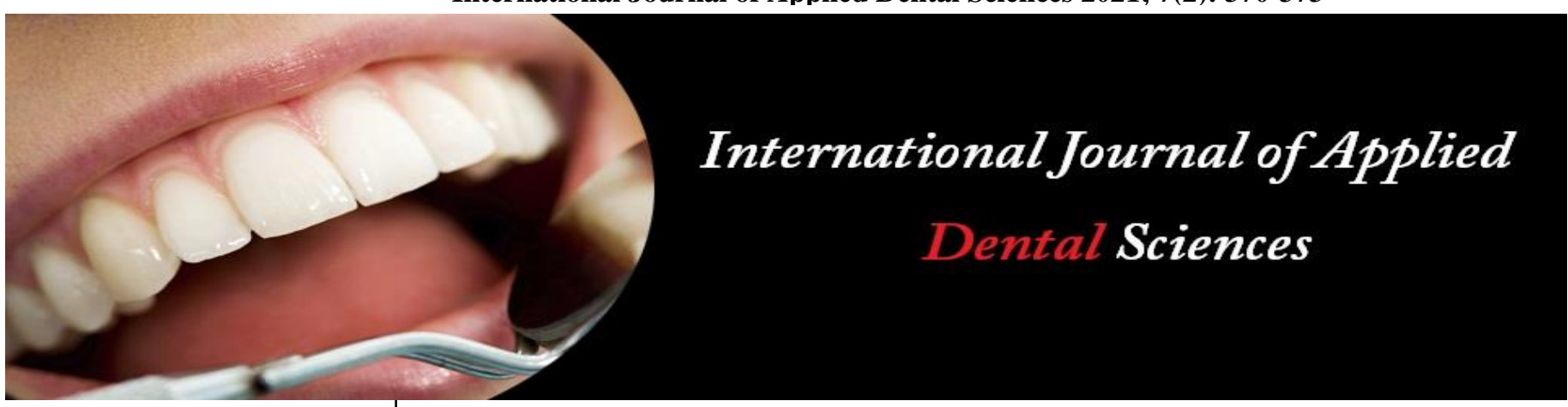

ISSN Print: 2394-7489

ISSN Online: 2394-7497

IJADS 2021; 7(2): 370-373

(C) 2021 IJADS

www.oraljournal.com

Received: 10-02-2021

Accepted: 12-30-2021

Shari SR

Junior Resident, Department of

Prosthodontics, Government

Dental College, Trivandrum,

Kerala, India

Harshakumar K

Professor, Department of

Prosthodontics, Government

Dental College, Trivandrum,

Kerala, India

Kavitha Janardanan

Assistant Professor, Department of Prosthodontics, Government

Dental College, Trivandrum,

Kerala, India
Corresponding Author:

Shari SR

Junior Resident, Department of

Prosthodontics, Government

Dental College, Trivandrum.

Kerala, India

\section{Fabrication of a hollow finger prosthesis using a three- piece mould technique: A case report}

\section{Shari SR, Harshakumar K and Kavitha Janardanan}

DOI: https://doi.org/10.22271/oral.2021.v7.i2f.1233

\section{Abstract}

Amputation of finger causes physical, psychological and economical harm to the patient. Restoration of the amputated part with a prosthesis made of silicone material can give a life like feel and protect the patient from social stigma. This case report describes a technique to fabricate a hollow finger prosthesis using Die-pin system. Hollow silicone finger prosthesis proved to be more comfortable and satisfying to the patient.

Keywords: amputation, hollow finger prosthesis, silicone finger prosthesis, three-piece mould, die-pin system

\section{Introduction}

Traumatic loss of fingers causes functional, psychological and aesthetic issues for an individual. The most common causes for the missing fingers are trauma and congenital absence/deformities ${ }^{[1-3]}$. Success of the finger prosthesis depends on the accuracy of designing wax pattern, selection of appropriate shade similar to the adjacent fingers, careful processing technique and final retention ${ }^{[4]}$.

Traditionally acrylic resin material was used for the fabrication of prosthesis, but this material lacks flexibility, hard to feel and has a tendency to stain easily. Nowadays different types of silicone material are available for the fabrication of prosthesis, which will give a natural look and soft feel like a skin. The artificial finger prosthesis should be comfortable and retentive then only prosthesis can satisfy the patient. In this case two digits are missing with a single stump which is slightly bulkier, hence the prosthesis needs to be hollowed out to reduce the weight and to increase the retention. This article describes a technique used to fabricate a hollow finger prosthesis, which creates a life like and natural feel to the patient

\section{Case history}

A $43 \mathrm{yr}$ old patient reported to the department of prosthodontics with a chief complaint of missing fingers on left hand. History revealed that he lost his fingers by an accident at a saw mill two years back. Examination showed that both index and middle finger were missing with only one residual stump present in the amputated area with no signs of discoloration or swelling (Fig. 1). On palpation, the stump was compressible in nature with no undercuts around it and had a sufficient length of $30 \mathrm{~mm}$ for the retention of the prosthesis.

\section{Treatment procedure}

A thin layer of petroleum jelly (Vaseline, Hindustan Lever, Mumbai, India) was applied over the patient's hand and he was instructed to place the fingers in a relaxed position and not to stretch while making an impression. A diagnostic impression of the left hand was made using irreversible hydrocolloid impression material (Tropicalgin, Zhermach Inc, California). The impression was poured using dental stone (Gyprock, Gujarat). For the fabrication of wax pattern, impression of another's person finger resembling the contour and size of the patient was made using irreversible hydrocolloid and poured in wax (Hindustan modelling wax no. 2, Hindustan dental products, Hyderabad). 
The wax pattern was hollowed out up to the desired length using hot wax spatula. Try-in of the wax pattern was done to assess the stability, adaptation and proper positioning in relation to the adjacent fingers (Fig. 2). Wax pattern along with the stump of the lost finger was cut from the model of the hand. In order to provide a good marginal fit for the prosthesis, $0.5 \mathrm{~mm}-1 \mathrm{~mm}$ of the stump was reduced with a bur. For making a three- piece mould, Die -Pin with sleeve (Pro-Fix, Renfert) was inserted along the long axis of the finger on the centre of the distal end of the stump (Fig. 3). A thin layer of petroleum jelly was applied over the stump. The wax pattern was placed on the stump and sealed with wax. A small rectangle shaped hole was created on the ventral aspect of the wax pattern (Fig. 4). The die stone (Gyprock, Gujarat) was mixed and taken in a syringe and injected through the hole into the wax pattern (Fig. 5). After complete pouring, the hole was sealed with wax. After setting of the die stone, the wax pattern was invested and dewaxed to get a three-piece mould (Fig. 6). The first part of the mould comprised of base part with the attached stump. The counter part of the flask forms the second part and the third part is the Detachable conical shaped part in between base and the counterpart.

Two different base shades were selected for ventral and dorsal aspect of the finger (Technovent, Intrinsic shade, Bridgent, UK) (Fig. 7). The detachable third part was removed before packing of the silicone. This facilitates easy packing of silicone material (A 2186, Factor II, Lakeside, USA) under the third piece of the mould without forming any voids. After packing the base shade silicone material for the ventral aspect, the third piece of the mould was inserted into the Die-Pin. The base shade silicone material for dorsal aspect was then packed into the counter mould, after which the flask was closed (Fig. 8). After the specified curing time the final hollow finger prosthesis was retrieved (Fig. 9). Final colour matching was carried out with the extrinsic colour (KT-199, Extrinsic colouration, Factor II, Lakeside, USA) for silicone material to get a natural look. The heat cured customised acrylic nail was attached to the finger prosthesis with the help of cyanoacrylate adhesive (Fevi-Kwik, Pidillite industries, Mumbai, India) (Fig. 10). An adjustable customised silver ring was used to increase the retention of the prosthesis and to mask the junction of the prosthesis and skin (Fig.11, 12).

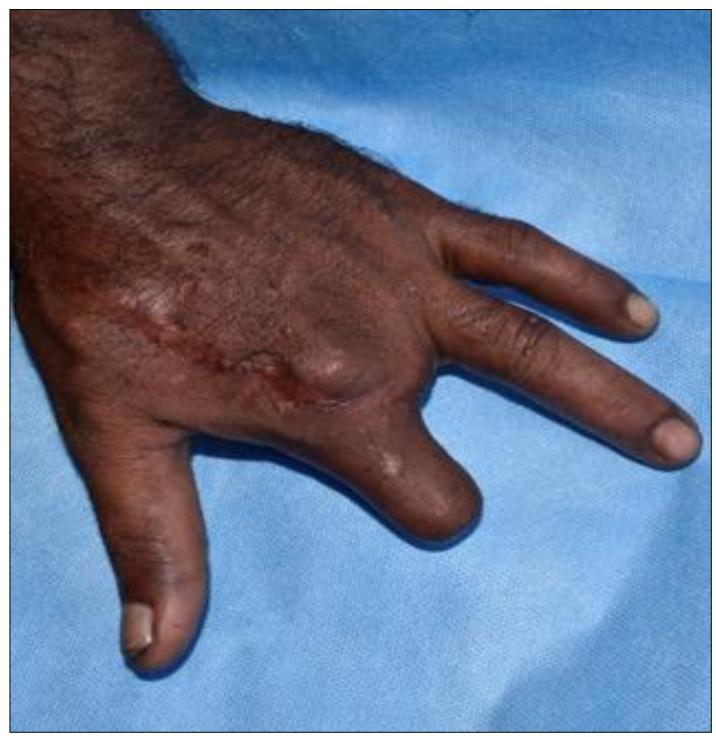

Fig 1: Pre-operative view of a hand with missing finger

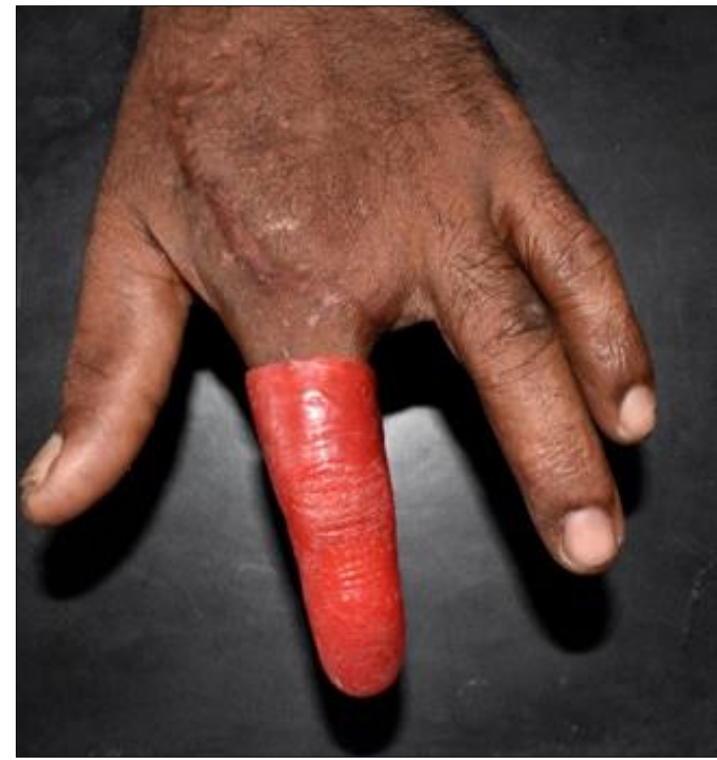

Fig 2: Wax pattern try-in

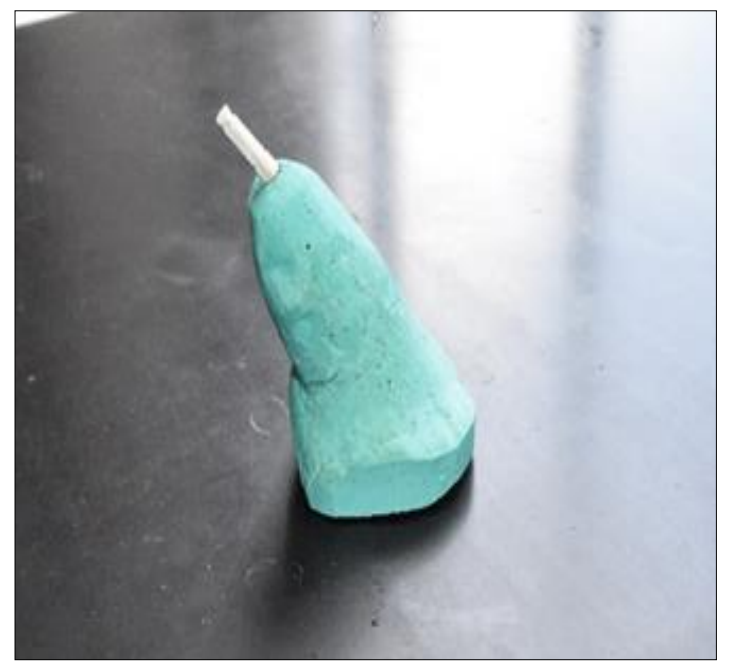

Fig 3: Die-pin with sleeve was inserted into a stone mould of the stump

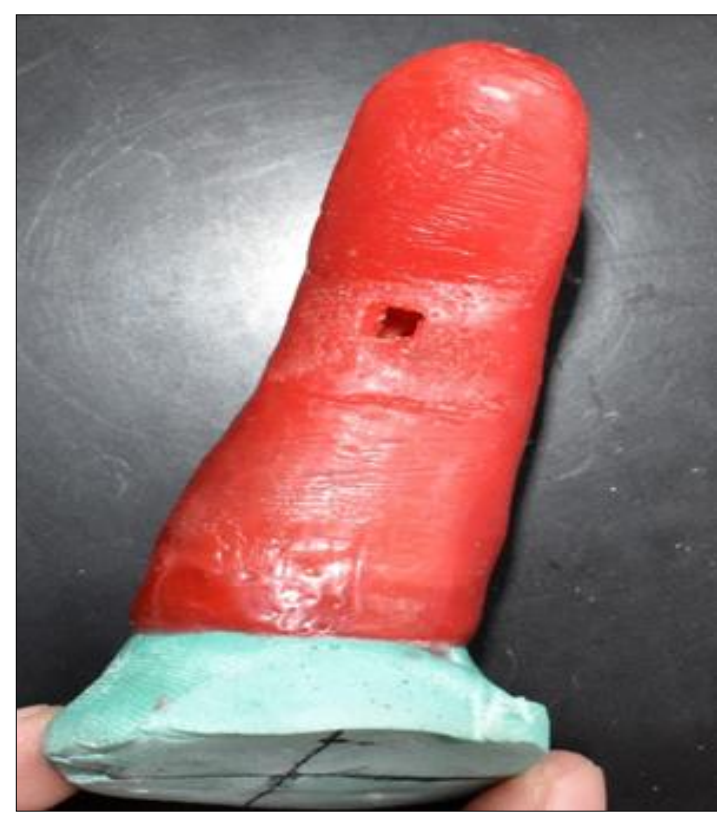

Fig 4: A rectangular hole was made on the ventral aspect of the wax pattern 


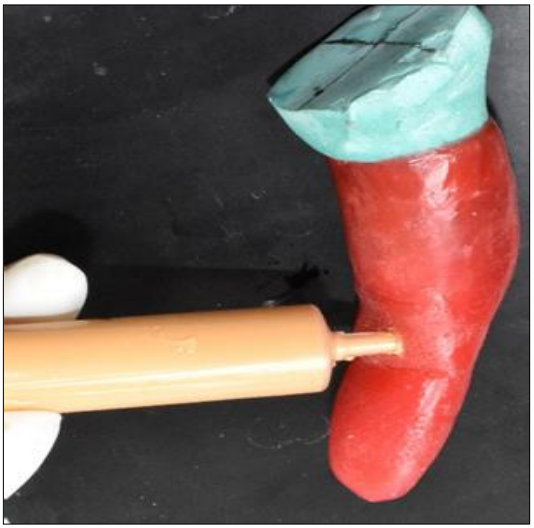

Fig 5: Die stone was injected through the hole

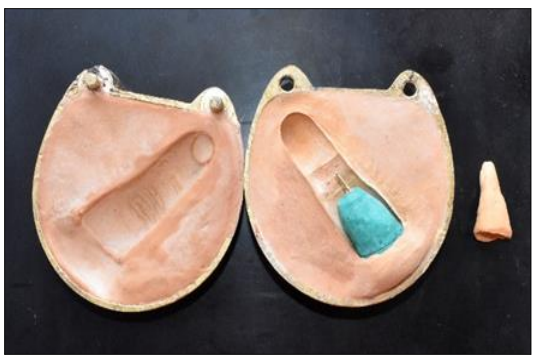

Fig 6: Three-piece mould

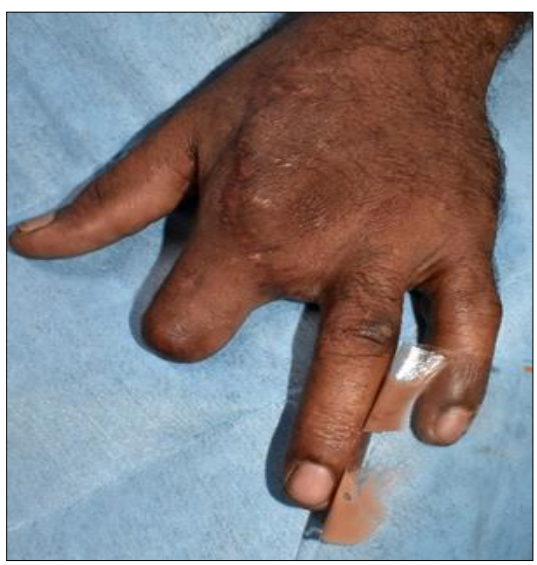

Fig 7: Shade selection

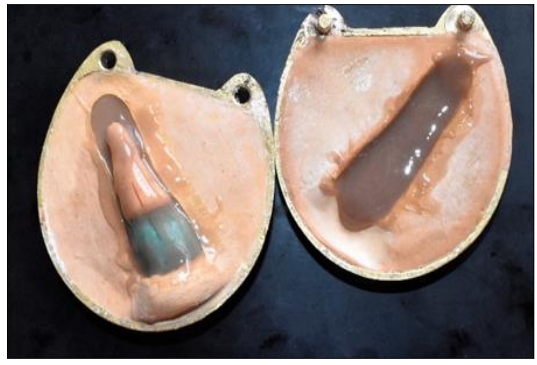

Fig 8: Silicone material was packed

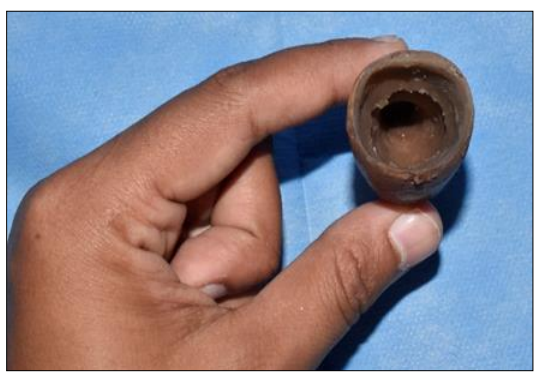

Fig 9: Hollow finger prosthesis

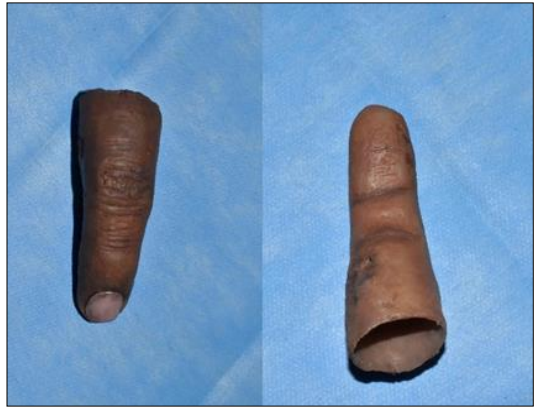

Fig 10: Final hollow finger prosthesis

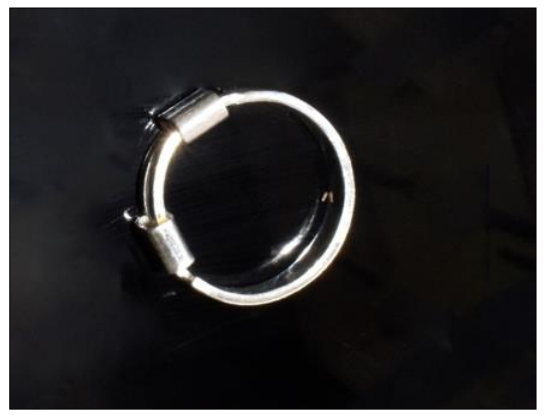

Fig 11: Customized adjustable silver ring

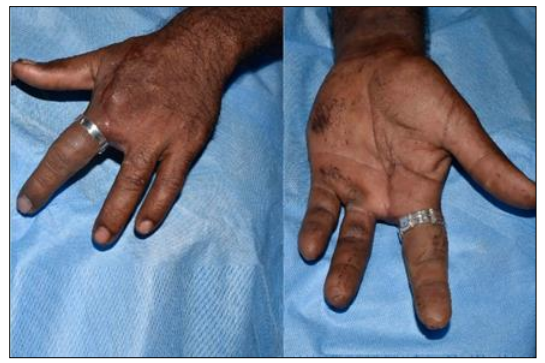

Fig 12: Post-operative view of hand with finger prosthesis

\section{Discussion}

Silicone prosthesis is excellent in terms of flexibility, colour matching, ease of fabrication and more lifelike appearance ${ }^{[5]}$. The case presented has long fingers and bulkier stump, so fabricating the finger prosthesis with full bulk will increase the weight of the prosthesis and cause discomfort for the patient during daily usage. The conical detachable third part of the mould helps in fabrication of a hollow prosthesis and easy colour customisation at the dorsal and ventral area of the finger ${ }^{[6]}$. The detachable third part helps to pack the silicone material in the mould without creating a large void in the ventral aspect of the prosthesis and uniform packing of the material in the base flask is possible.

Michael and Buckner ${ }^{[7]}$ suggested a method of scoring the model to increase the fit of the prosthesis which was based on the presence of compressible tissues in the residual stump. In this case, compressibility of tissue was present on the ventral aspect of the residual stump. Therefore, scoring was performed and glove type prosthesis was chosen for the patient.

\section{Conclusion}

The loss of finger can affect the individual physically, emotionally and psychologically. Artificial finger prosthesis can camouflage the defect created due to the loss of finger. By reducing the bulk of the prosthesis will give comfort to the patient. Here we used a three -piece mould technique with the help of a Die- Pin system to make the prosthesis hollow and it was successful. 


\section{References}

1. Pillet J. The aesthetic hand prosthesis. Orthop Clin North Am 1981;12:961-9.

2. Beasley RW. General considerations in managing upper limb amputations. Orthop Clin North Am 1981;12:743-9.

3. Michael J. Partial-hand amputation: prosthetic and orthotic management. Atlas Limb Prosthet Surg Prosthet Rehabil Princ CV Mosby St Louis 1992, 217-226.

4. Herring HW, Rommerdale EH. Prosthetic Finger Retention: A New Approach. Orthot Prosthet 1983;3:2830.

5. Beumer J III, Curtis TA, Firtell DN. Maxillofacial rehabilitation, prosthodontic and surgical considerations. London: CV Mosby Company 1979, 528.

6. Ajay Jain, Fraser Walker S, Sridevi Ugrappa, Sumit Makkad, Vijay Kumar Ugrappa. Three-Part mould technique for fabrication of hollow thumb prosthesis: A case report. Prosthetics and Orthotics International 2016;40:756-762.

7. Michael JW, Buckner H. Options for finger prosthesis. J Prosthet Orthot 1994;6:10-19. 